Supporting information for:

\title{
A hydrogen bonding-modulated molecular rotor: environmental effect in the conformational stability of peptidomimetic macrocyclic cyclophanes.
}

\author{
Ignacio Alfonso, M. Isabel Burguete and Santiago V. Luis*
}

Departamento de Química Inorgánica y Orgánica, UAMOA, Universidad Jaume I, Campus del Riu Sec, Avenida Sos Baynat, s/n, E-12071, Castellón (Spain).

$$
\text { luiss@qio.uji.es }
$$

Table of contents:

${ }^{1} \mathrm{H}^{1} \mathrm{H}$ COSY spectrum of $\mathbf{1 a}\left(\mathrm{CDCl}_{3}, 500 \mathrm{MHz}, 303 \mathrm{~K}\right) \quad \mathrm{S}-2$

${ }^{1} \mathrm{H}^{1} \mathrm{H}$ COSY spectrum of $\mathbf{1 b}\left(\mathrm{CDCl}_{3}, 500 \mathrm{MHz}, 303 \mathrm{~K}\right) \quad$ S-3

${ }^{1} \mathrm{H}^{1} \mathrm{H}$ COSY spectrum of $\mathbf{2 a}\left(\mathrm{CDCl}_{3}, 500 \mathrm{MHz}, 303 \mathrm{~K}\right) \quad$ S-4

${ }^{1} \mathrm{H}{ }^{1} \mathrm{H}$ COSY spectrum of $\mathbf{2 b}\left(\mathrm{CDCl}_{3}, 500 \mathrm{MHz}, 303 \mathrm{~K}\right) \quad$ S-5

${ }^{1} \mathrm{H}^{1} \mathrm{H}$ COSY spectrum of $\mathbf{3 a}\left(\mathrm{CDCl}_{3}, 500 \mathrm{MHz}, 303 \mathrm{~K}\right) \quad$ S-6

${ }^{1} \mathrm{H}^{1} \mathrm{H}$ COSY spectrum of $\mathbf{3 b}\left(\mathrm{CDCl}_{3}, 500 \mathrm{MHz}, 303 \mathrm{~K}\right) \quad$ S-7

VT-NMR and Eyring/Arrhenius plots of $1 \mathbf{a}\left(300 \mathrm{MHz}, \mathrm{DMSO}-d_{6}\right)$

S-8

VT-NMR and Eyring/Arrhenius plots of $\mathbf{1 b}\left(300 \mathrm{MHz}\right.$, DMSO- $\left.d_{6}\right)$

S-9

VT-NMR and Eyring/Arrhenius plots of $\mathbf{2 a}\left(500 \mathrm{MHz}, \mathrm{CDCl}_{3}\right) \quad$ S-10

$\begin{array}{ll}\text { VT-NMR and Eyring/Arrhenius plots of } \mathbf{2 b}\left(500 \mathrm{MHz}, \mathrm{CDCl}_{3}\right) & \text { S-11 }\end{array}$

VT-NMR and Eyring/Arrhenius plots of $2 \mathbf{a}\left(500 \mathrm{MHz}, \mathrm{CD}_{3} \mathrm{COCD}_{3}: \mathrm{CD}_{3} \mathrm{OD}\right.$ 9:1)

S-12

Plot of amide $\mathrm{N} H$ chemical shifts vs temperature

Superimposed ten lowest energy conformations for peptidomimetic cyclophanes 1a,b-2a,b

S-13

Chemical shifts and difference produced by the aromatic side chain on $\mathbf{1 a , b - 2 a , b}$

S-14

Cartesian coordinates of the minimum of $\mathbf{1 a}$

S-14

Cartesian coordinates of the minimum of $\mathbf{1 b}$

S-15 
Cartesian coordinates of the minimum of $\mathbf{2 a}$

S-17

Cartesian coordinates of the minimum of $\mathbf{2 b}$

S-18

Cartesian coordinates of the transition state of $\mathbf{1 a}$

S-20 
${ }^{1} \mathrm{H}^{1} \mathrm{H}$ COSY spectrum of $1 \mathrm{a}\left(\mathrm{CDCl}_{3}, 500 \mathrm{MHz}, 303 \mathrm{~K}\right)$.

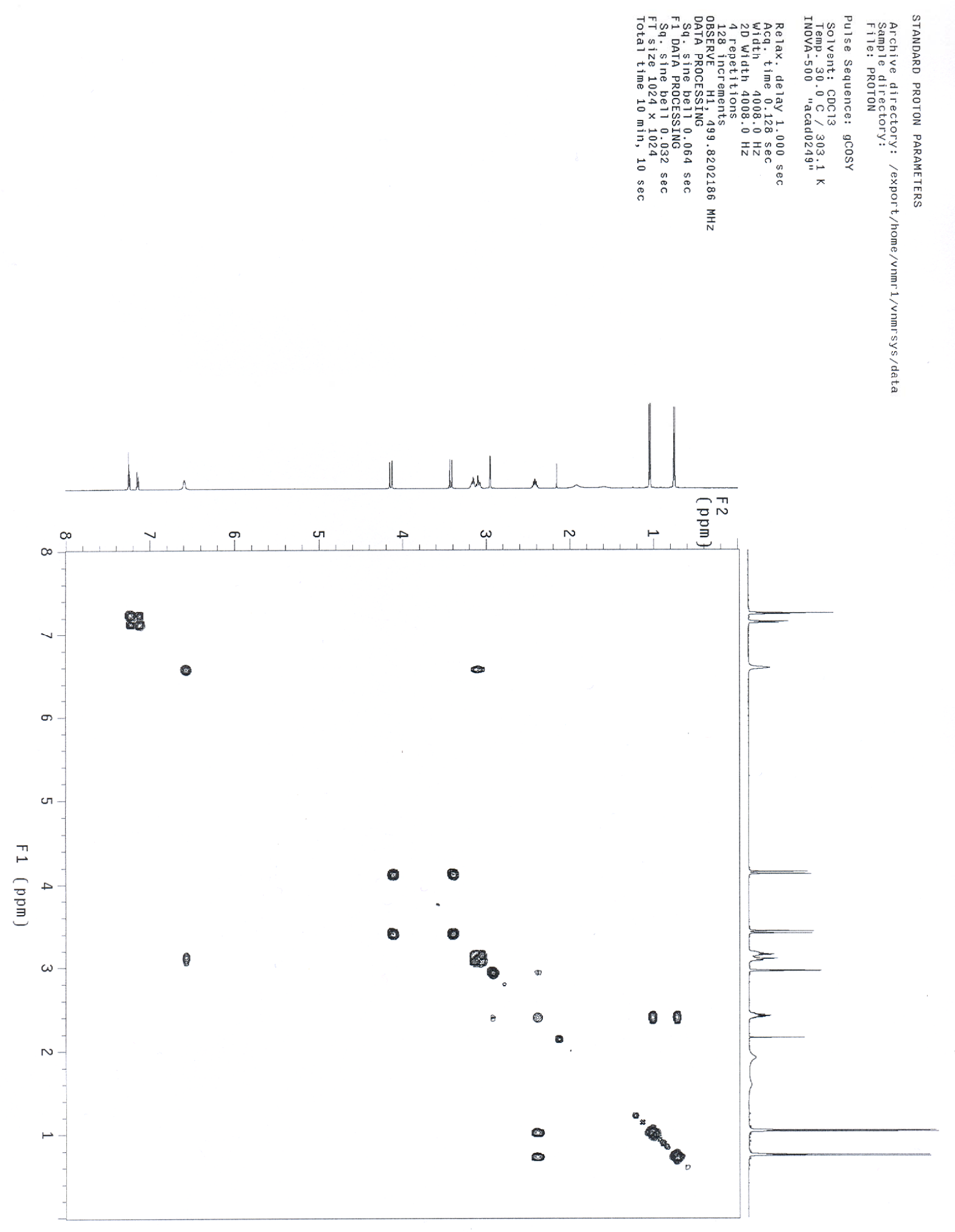


${ }^{1} \mathrm{H}^{1} \mathrm{H}$ COSY spectrum of $1 \mathrm{~b}\left(\mathrm{CDCl}_{3}, 500 \mathrm{MHz}, 303 \mathrm{~K}\right)$.

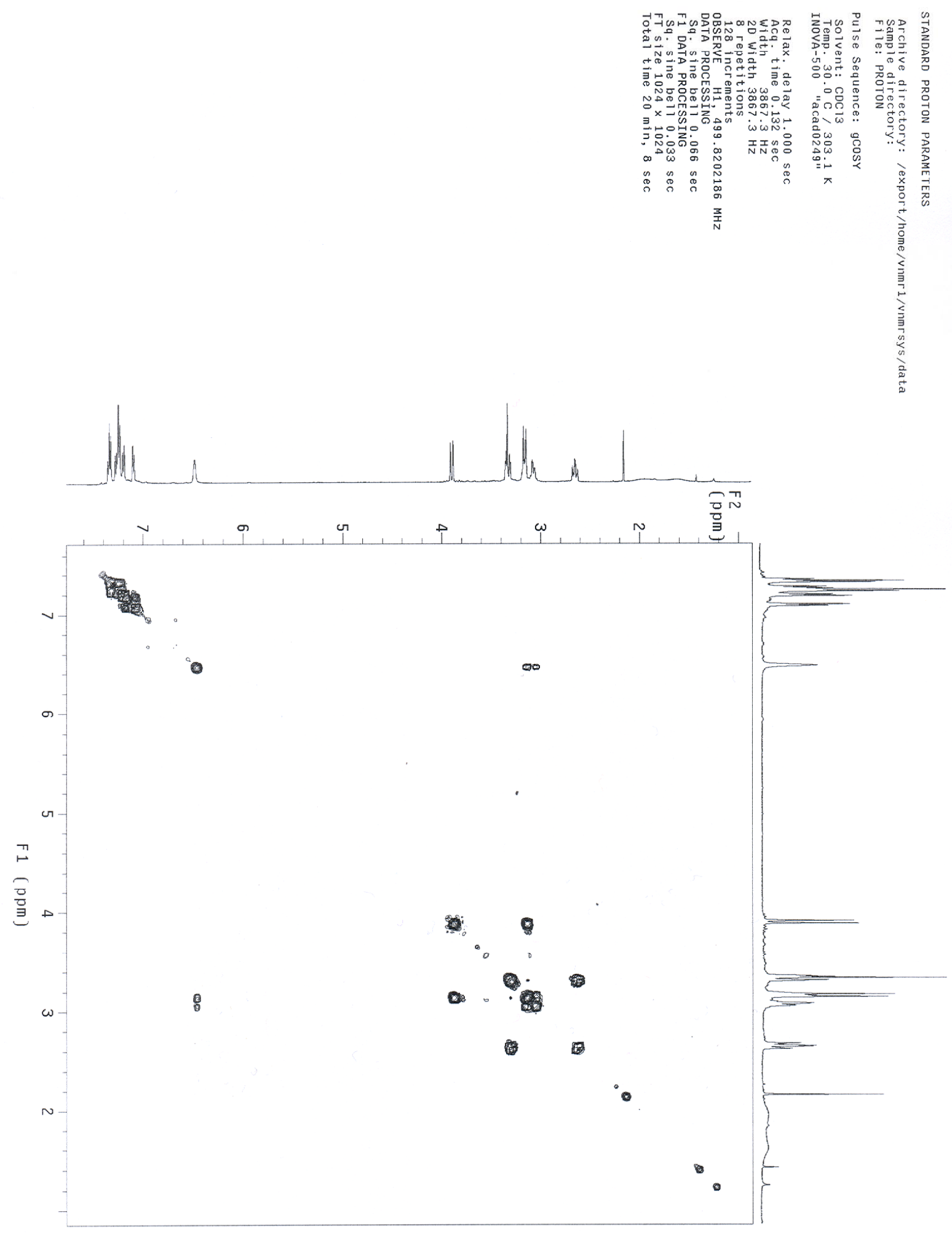


${ }^{1} \mathrm{H}^{1} \mathrm{H}$ COSY spectrum of $2 \mathrm{a}\left(\mathrm{CDCl}_{3}, 500 \mathrm{MHz}, 303 \mathrm{~K}\right)$.

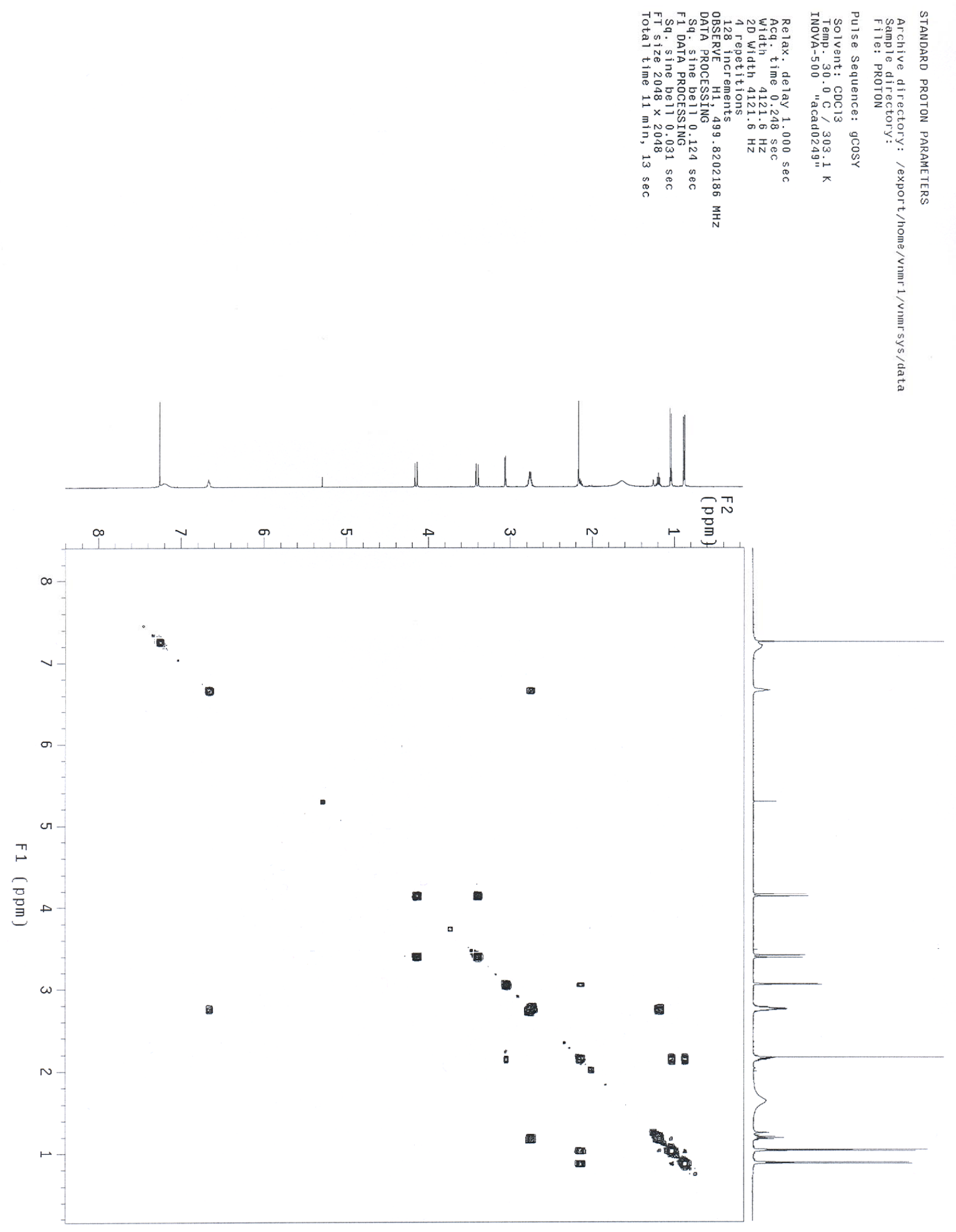


${ }^{1} \mathrm{H}^{1} \mathrm{H}$ COSY spectrum of $2 \mathrm{~b}\left(\mathrm{CDCl}_{3}, 500 \mathrm{MHz}, 303 \mathrm{~K}\right)$.

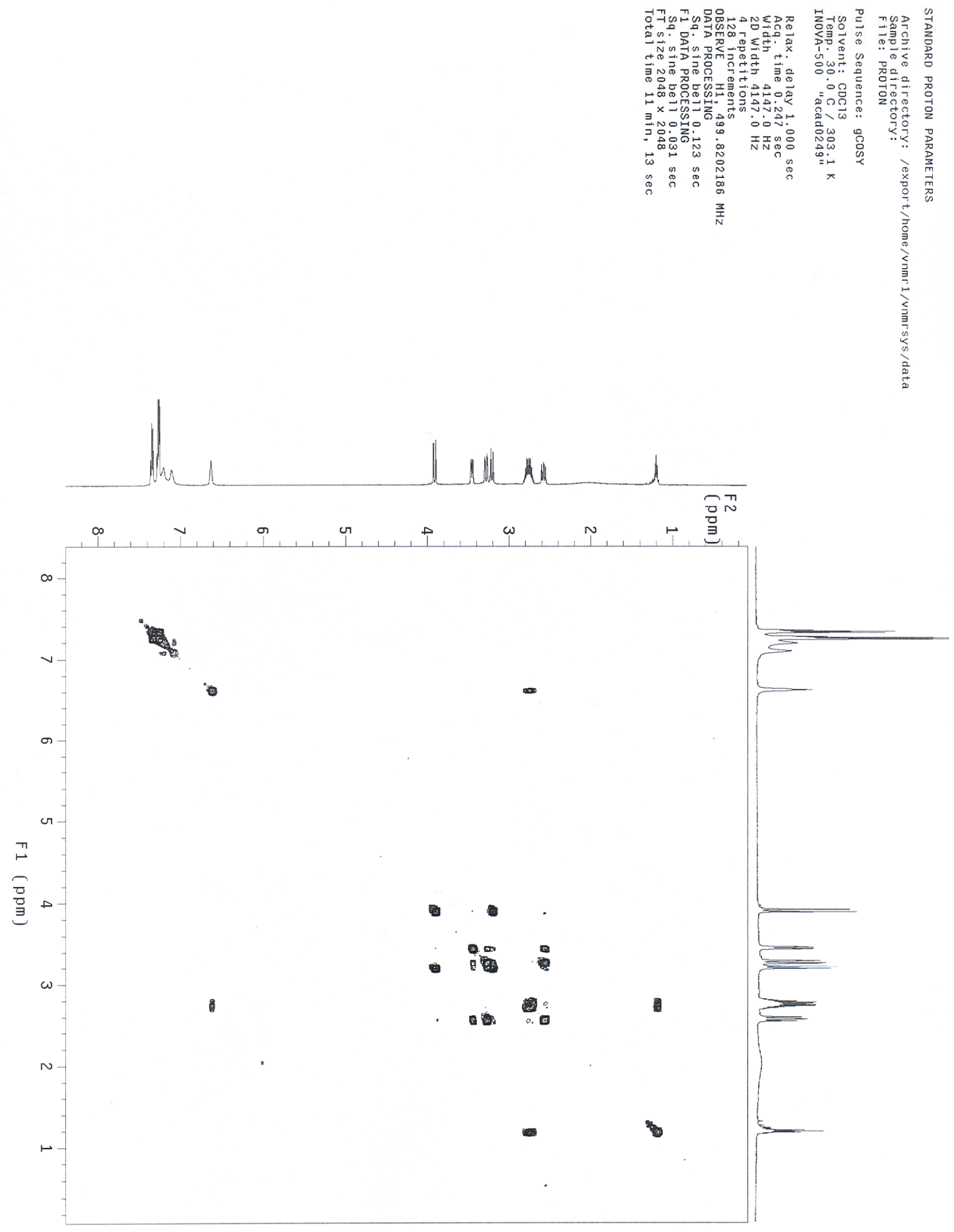


${ }^{1} \mathrm{H}^{1} \mathrm{H}$ COSY spectrum of $3 \mathrm{a}\left(\mathrm{CDCl}_{3}, 500 \mathrm{MHz}, 303 \mathrm{~K}\right)$.

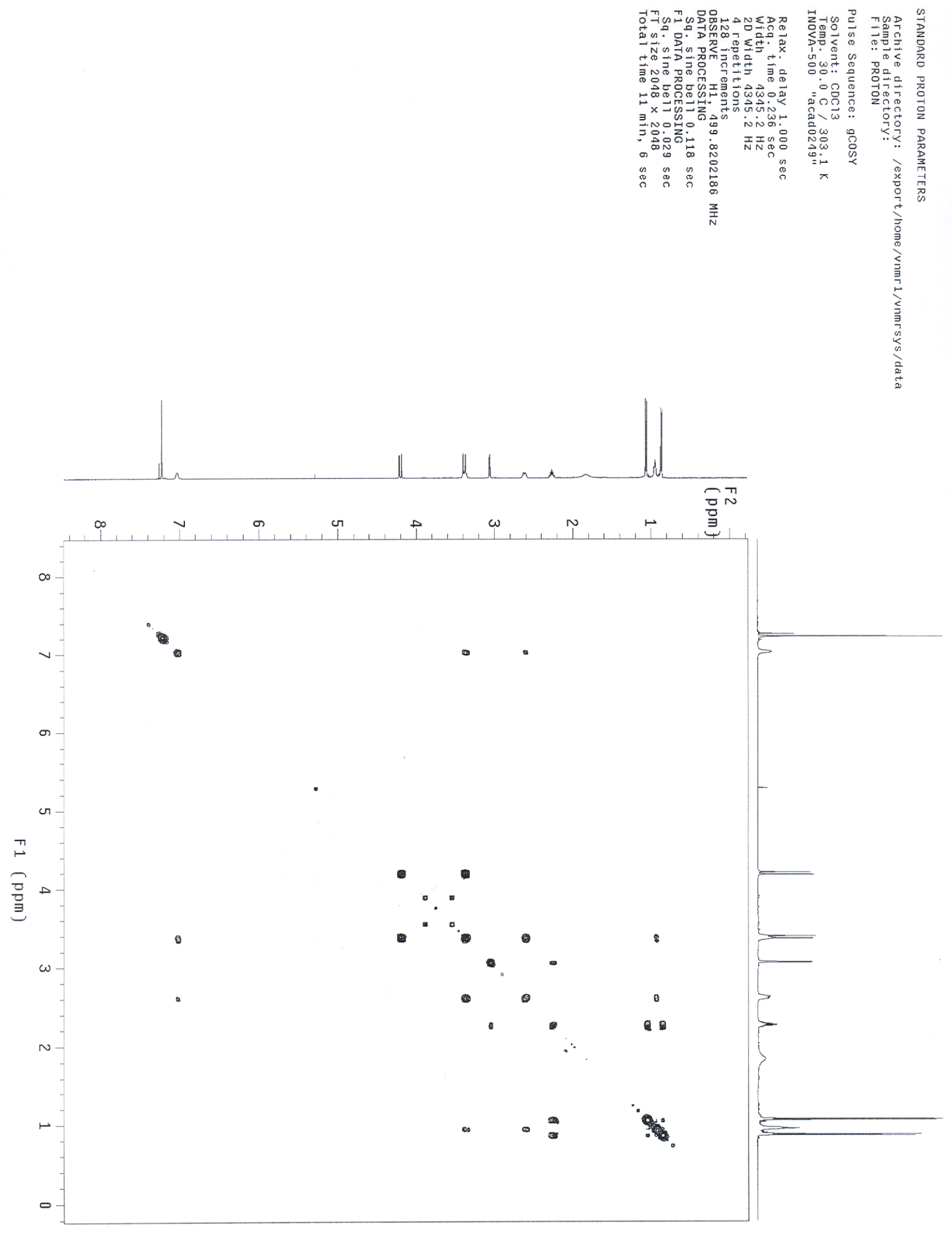


${ }^{1} \mathrm{H}^{1} \mathrm{H}$ COSY spectrum of $3 \mathrm{~b}\left(\mathrm{CDCl}_{3}, 500 \mathrm{MHz}, 303 \mathrm{~K}\right)$.

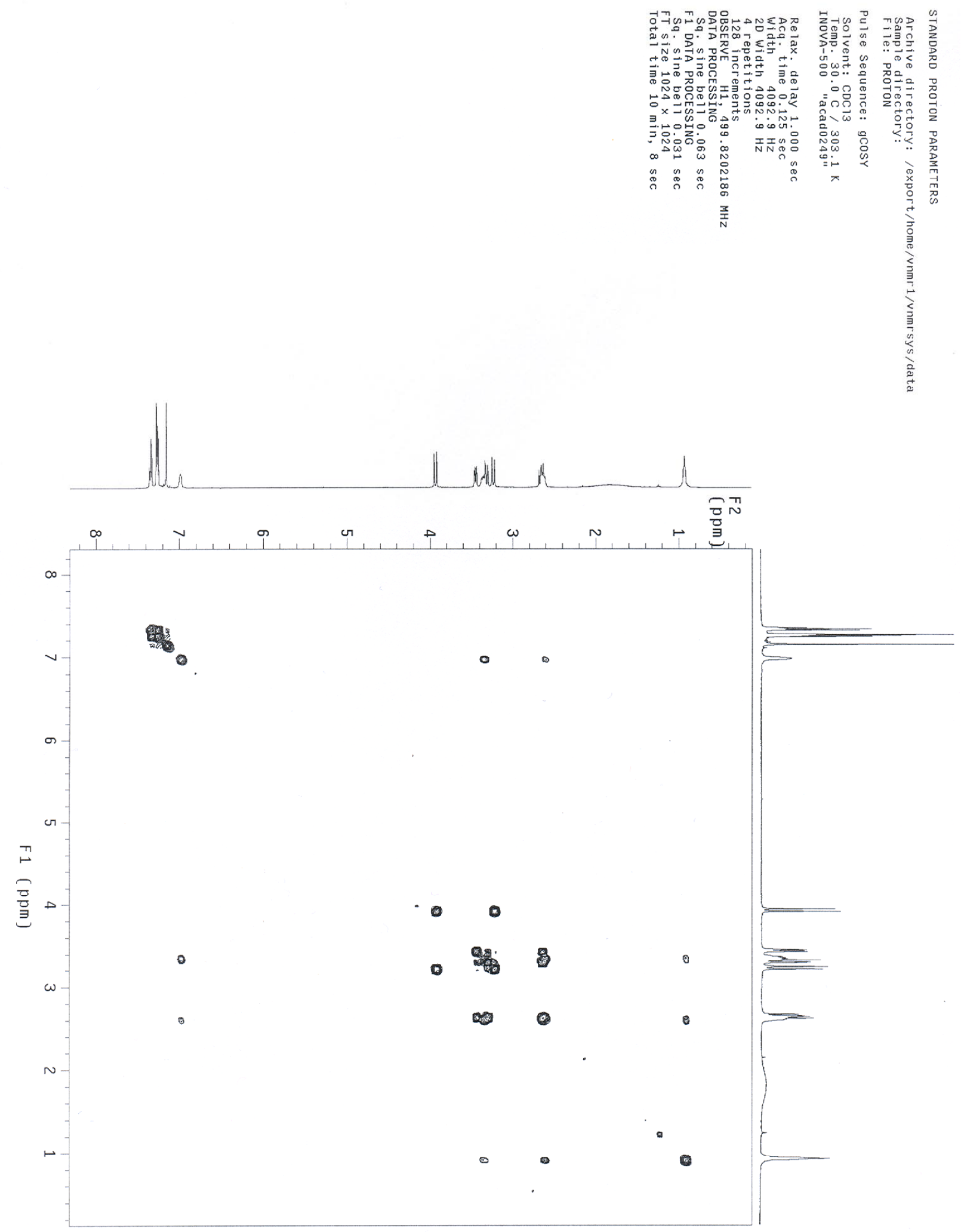


Experimental (upper trace) and simulated (lower trace) spectra of 1a (DMSO- $\left.d_{6}, 300 \mathrm{MHz}\right)$ at different temperatures. Bottom: Eyring and Arrhenius plots for the corresponding analysis.
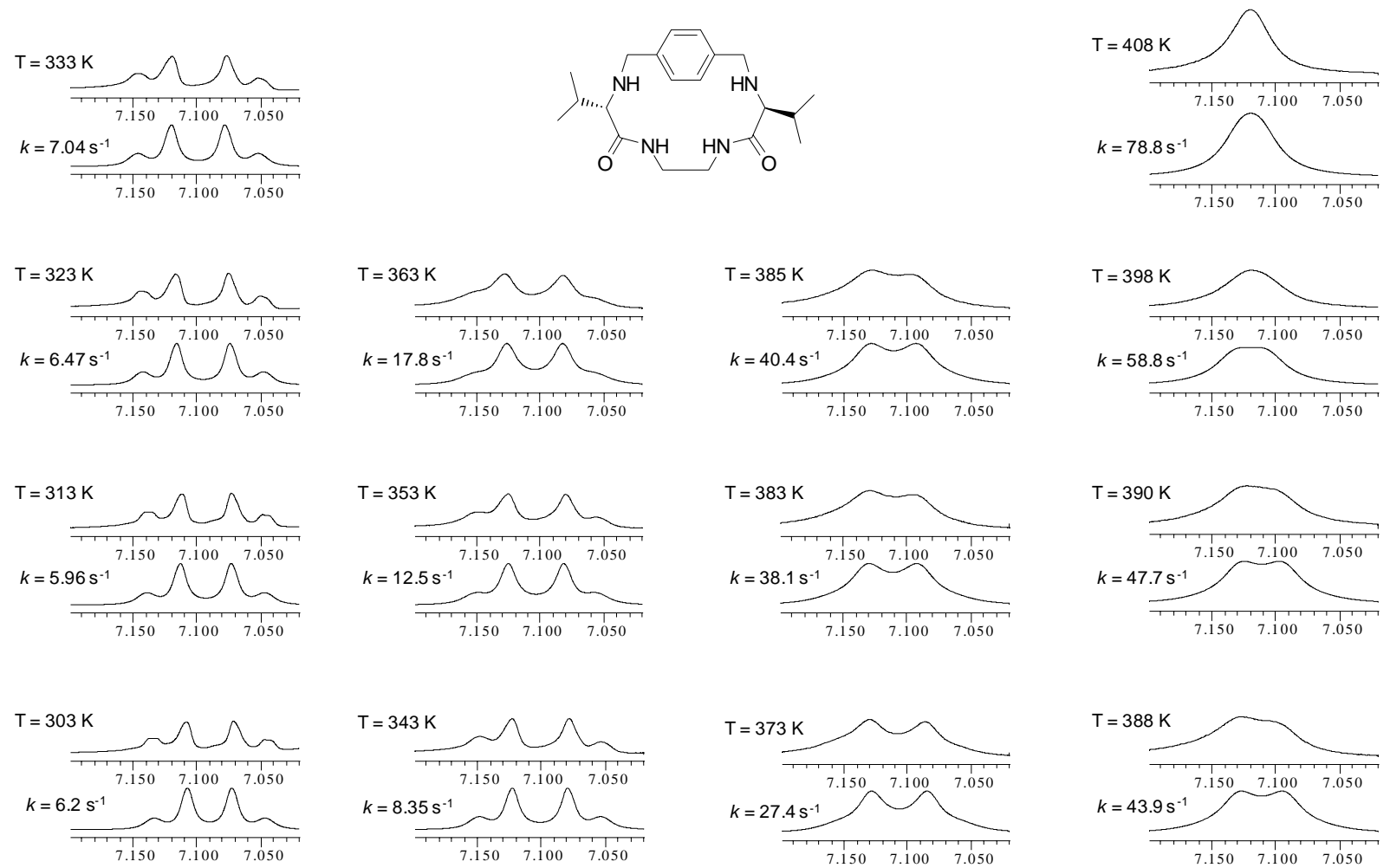

Eyring plot

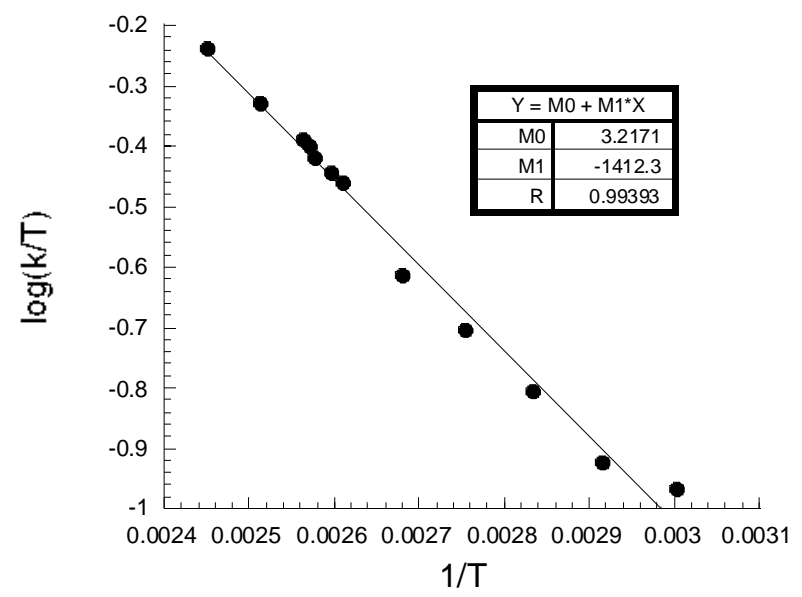

$\Delta H^{\#}=6.45 \mathrm{Kcal} / \mathrm{mol}$

$\Delta S^{\#}=-33.59 \mathrm{cal} /(\mathrm{K} \mathrm{mol})$

$\Delta G^{\#}(298 \mathrm{~K})=16.46 \mathrm{Kcal} / \mathrm{mol}$

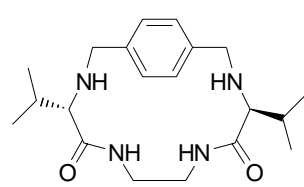

\section{Arrhenius plot}

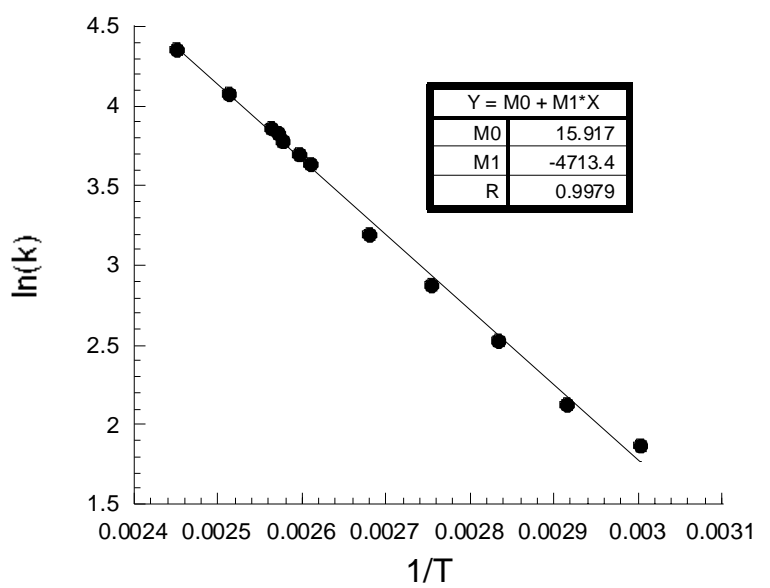

Ea $($ Arrhenius $)=9.71 \mathrm{Kcal} / \mathrm{mol}$ 
Experimental (upper trace) and simulated (lower trace) spectra of $\mathbf{1 b}\left(\mathrm{DMSO}-d_{6}, 300 \mathrm{MHz}\right)$ at different temperatures. Bottom: Eyring and Arrhenius plots for the corresponding analysis.
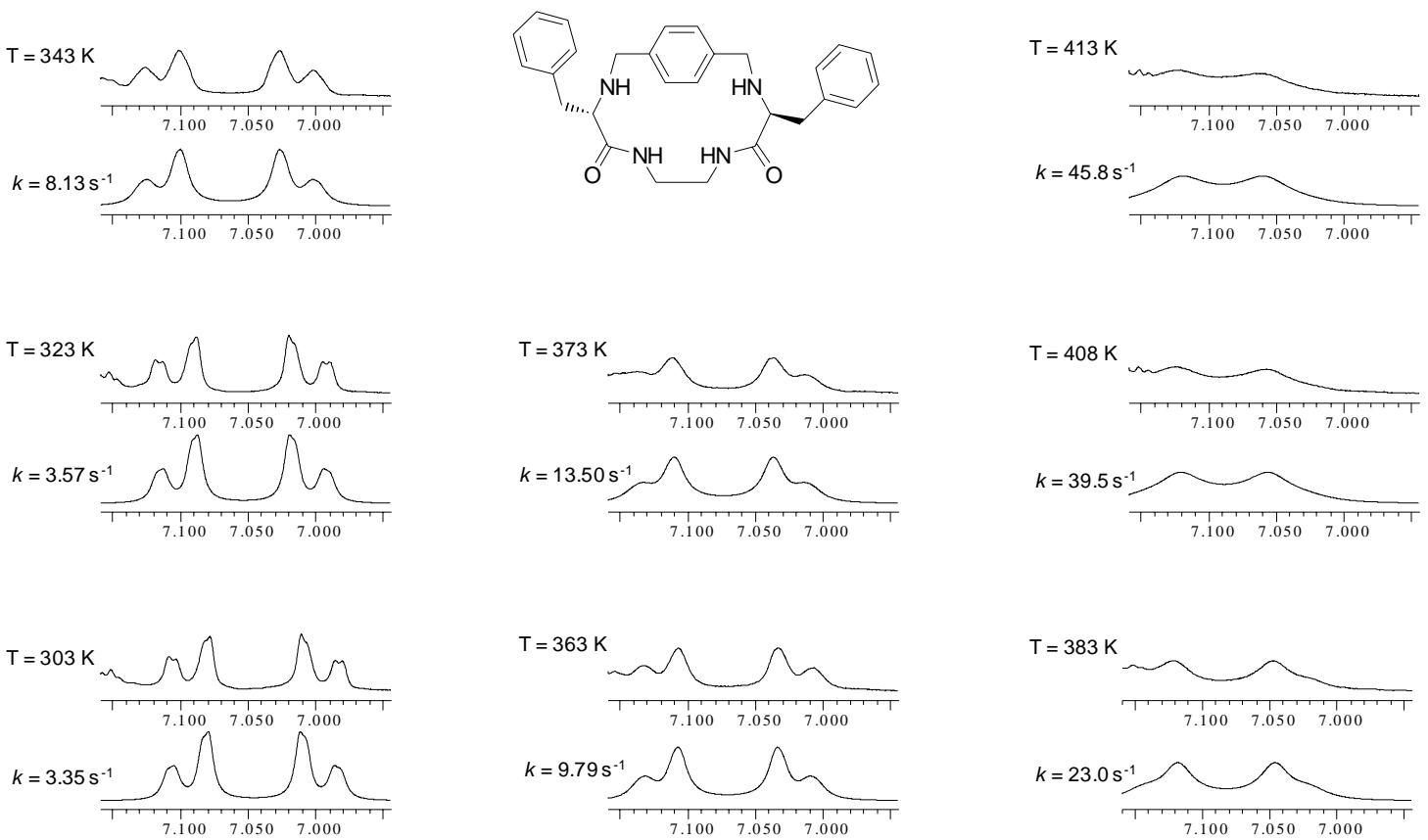

Eyring plot

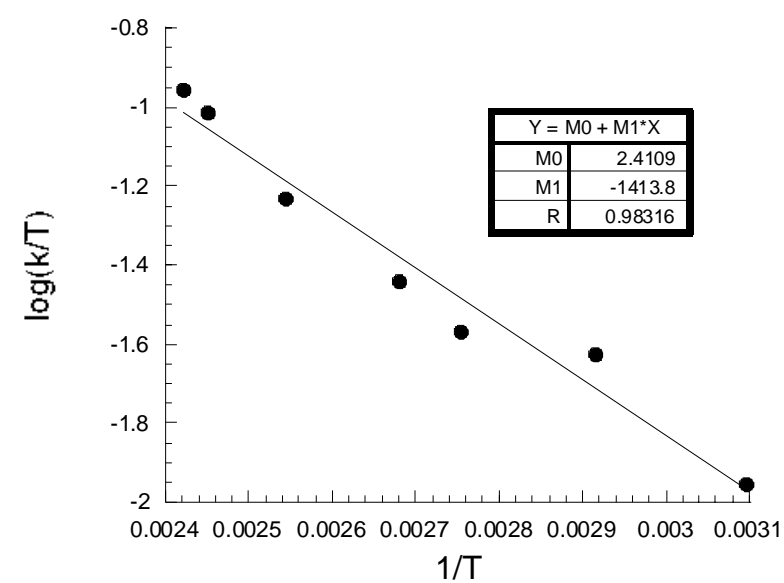

$\Delta H^{\#}=6.46 \mathrm{Kcal} / \mathrm{mol}$

$\Delta S^{\#}=-36.14 \mathrm{cal} /(\mathrm{K} \mathrm{mol})$

$\Delta G^{\#}(298 \mathrm{~K})=17.23 \mathrm{Kcal} / \mathrm{mol}$
Arrhenius plot

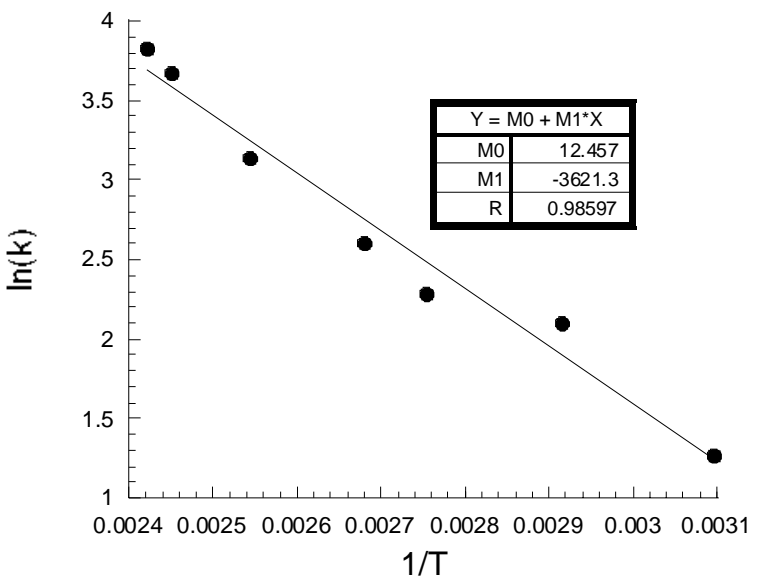

Ea $($ Arrhenius $)=7.23 \mathrm{Kcal} / \mathrm{mol}$ 
Experimental (upper trace) and simulated (lower trace) spectra of $\mathbf{2 a}\left(\mathrm{CDCl}_{3}, 500 \mathrm{MHz}\right)$ at different temperatures. Bottom: Eyring and Arrhenius plots for the corresponding analysis.
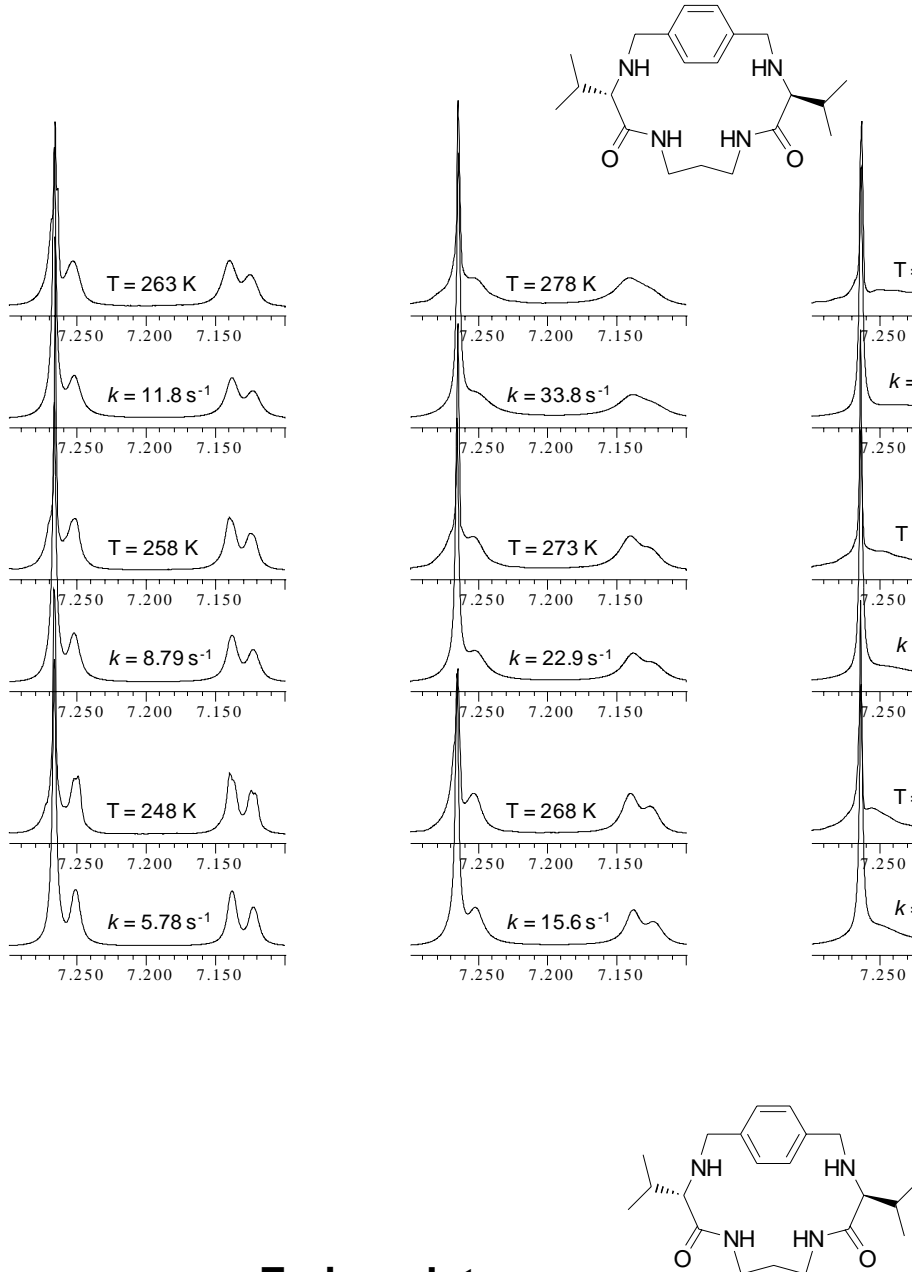

Eyring plot

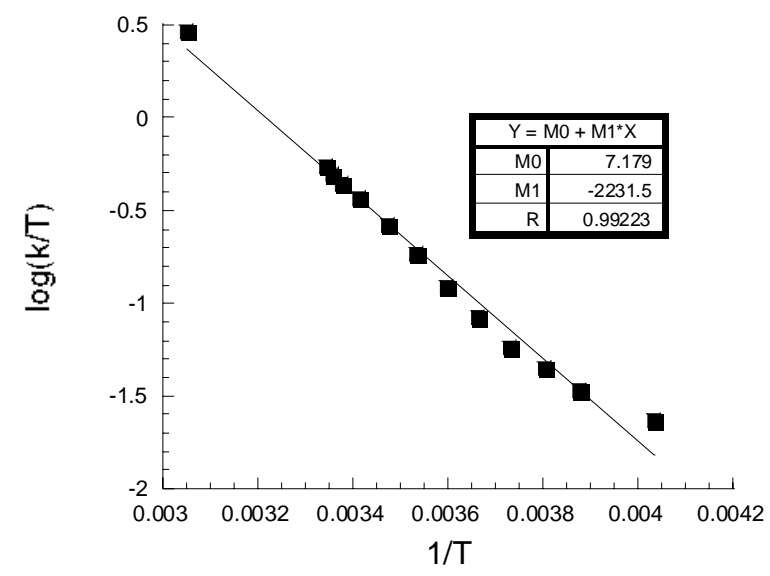

$\Delta H^{\#}=10.20 \mathrm{Kcal} / \mathrm{mol}$

$\Delta S^{\#}=-14.35 \mathrm{cal} /(\mathrm{K} \mathrm{mol})$

$\Delta G^{\#}(298 \mathrm{~K})=14.48 \mathrm{Kcal} / \mathrm{mol}$

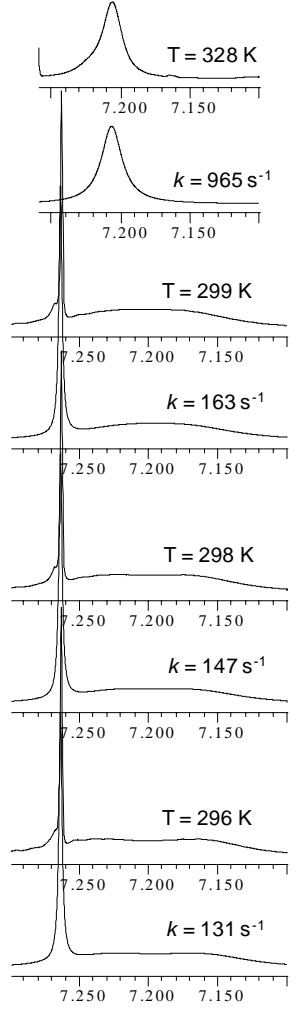

Arrhenius plot

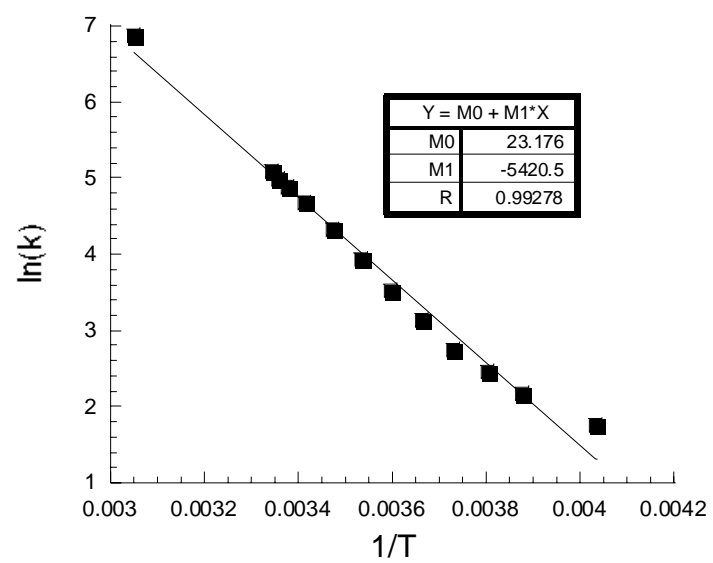

Ea $($ Arrhenius $)=10.82 \mathrm{Kcal} / \mathrm{mol}$ 
Experimental (upper trace) and simulated (lower trace) spectra of $\mathbf{2 b}\left(\mathrm{CDCl}_{3}, 500 \mathrm{MHz}\right)$ at different temperatures. Bottom: Eyring and Arrhenius plots for the corresponding analysis.
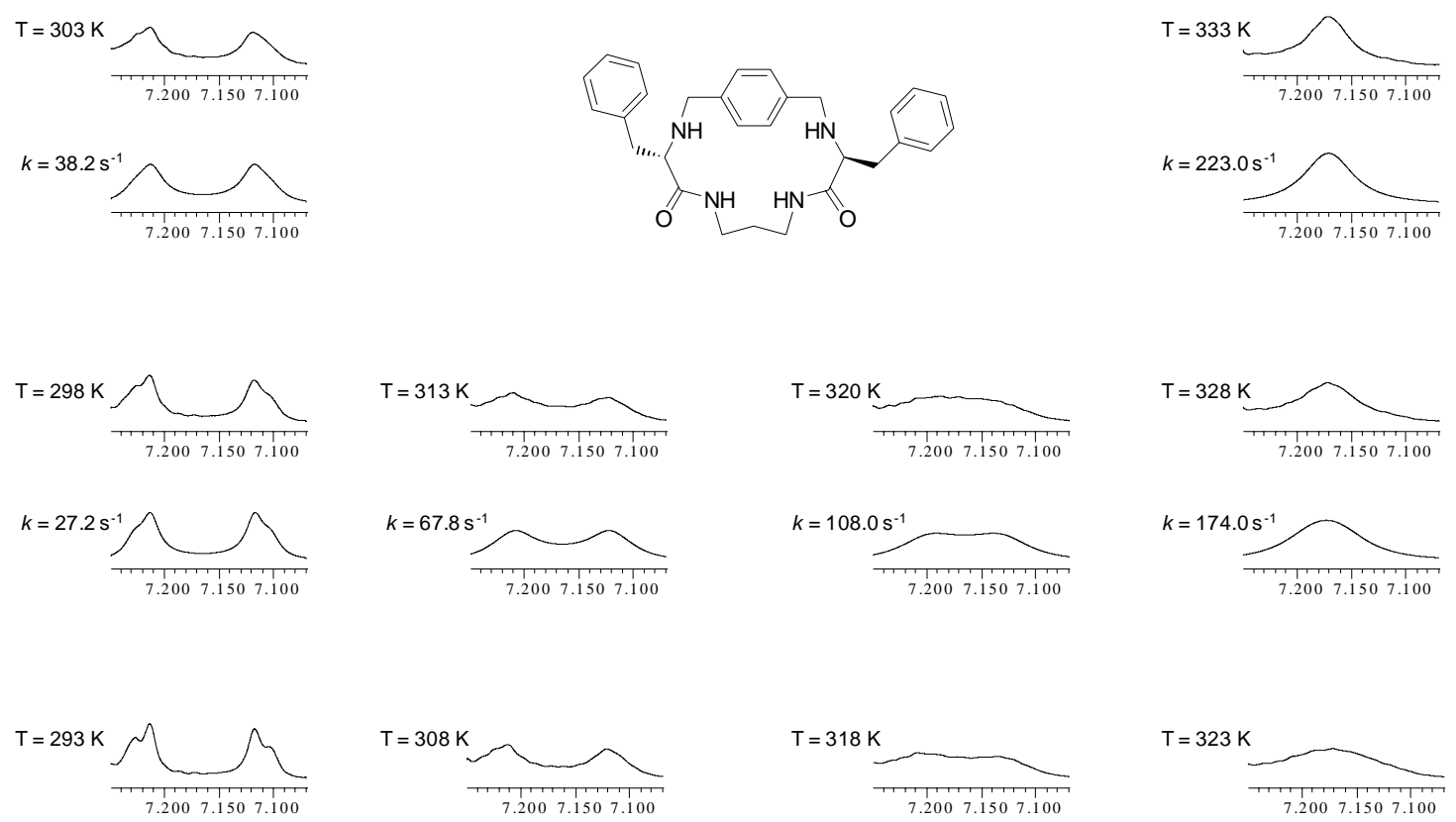

$\mathrm{T}=323 \mathrm{~K}$
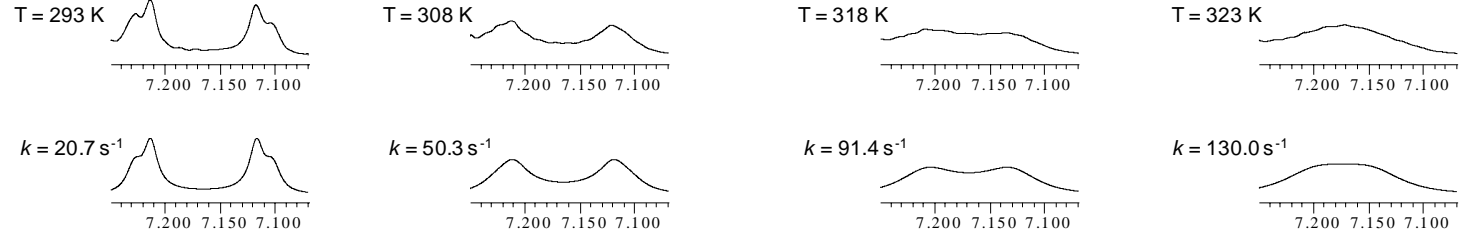

Eyring plot

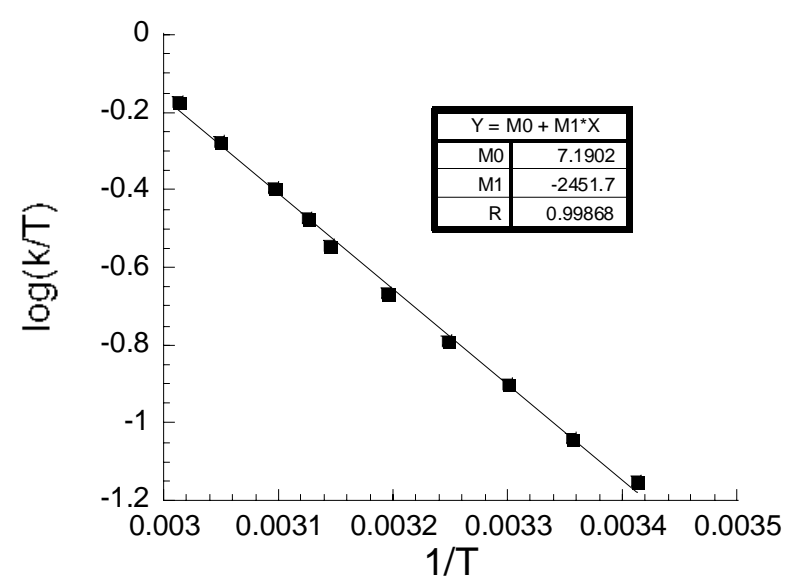

$\Delta H^{\#}=11.39 \mathrm{Kcal} / \mathrm{mol}$

$\Delta S^{\#}=-14.30 \mathrm{cal} /(\mathrm{K} \mathrm{mol})$ $\Delta G^{\#}(298 \mathrm{~K})=15.65 \mathrm{Kcal} / \mathrm{mol}$

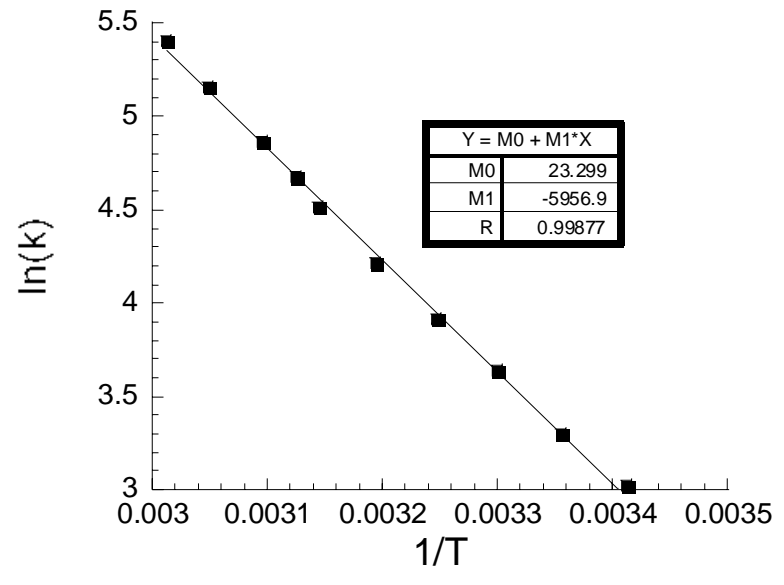

Ea $($ Arrhenius $)=11.89 \mathrm{Kcal} / \mathrm{mol}$ 
Experimental (upper trace) and simulated (lower trace) spectra of 2a (acetone- $d_{6}:$ methanol- $d_{4}$ 9:1, $500 \mathrm{MHz}$ ) at different temperatures. Bottom: Eyring and Arrhenius plots for the corresponding analysis.
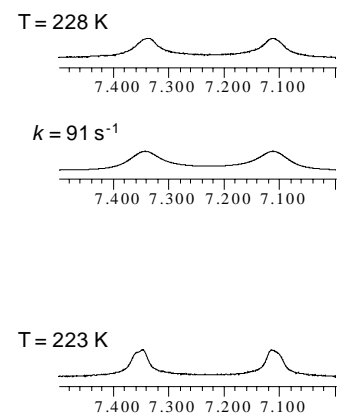

$k=56.7 \mathrm{~s}^{-1}$

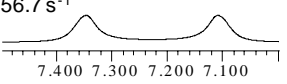

$\mathrm{T}=218 \mathrm{~K}$

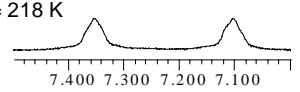

$k=43.5 \mathrm{~s}^{-1}$

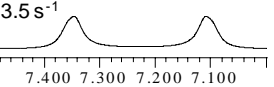

$\mathrm{T}=236 \mathrm{~K}$

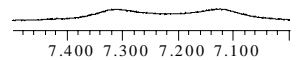

$k=179 \mathrm{~s}^{-1}$

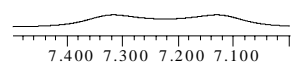

$\mathrm{T}=233 \mathrm{~K}$

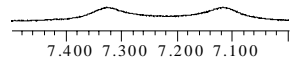

$k=140 \mathrm{~s}^{-1}$

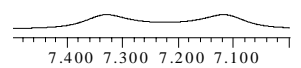

$\mathrm{T}=240 \mathrm{~K}$

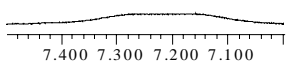

$k=259 \mathrm{~s}^{-1}$

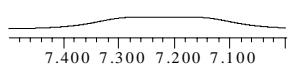

$\mathrm{T}=238 \mathrm{~K}$

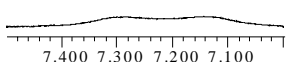

$k=216 s^{-1}$

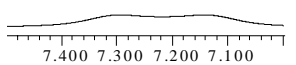

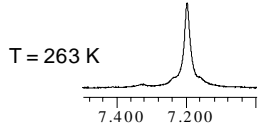
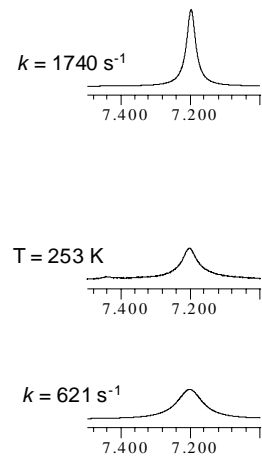

$\mathrm{T}=243 \mathrm{~K}$

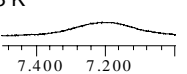

$k=308 \mathrm{~s}^{-1}$

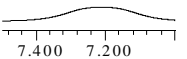

Eyring plot

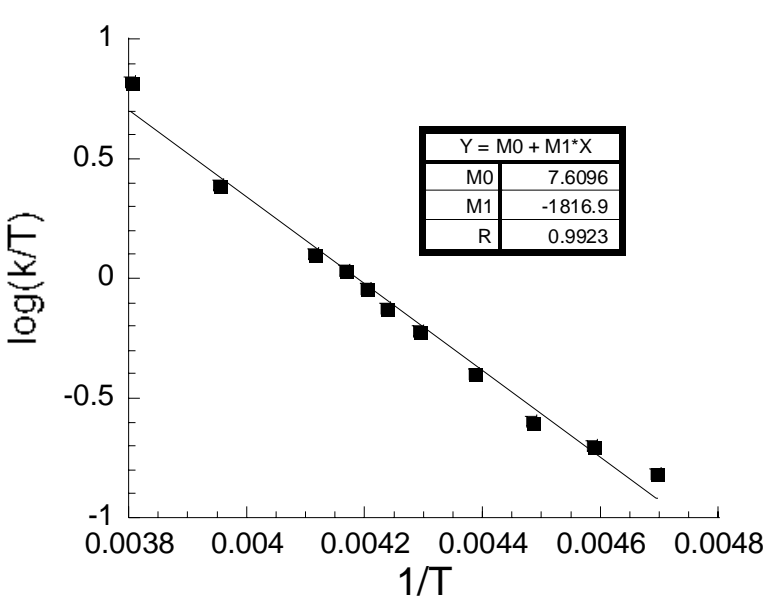

$\Delta H^{\#}=8.30 \mathrm{Kcal} / \mathrm{mol}$

$\Delta S^{\#}=-12.39 \mathrm{cal} /(\mathrm{K} \mathrm{mol})$

$\Delta G^{\#}(298 \mathrm{~K})=11.19 \mathrm{Kcal} / \mathrm{mol}$

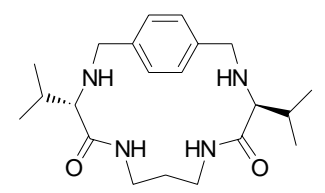

Arrhenius plot

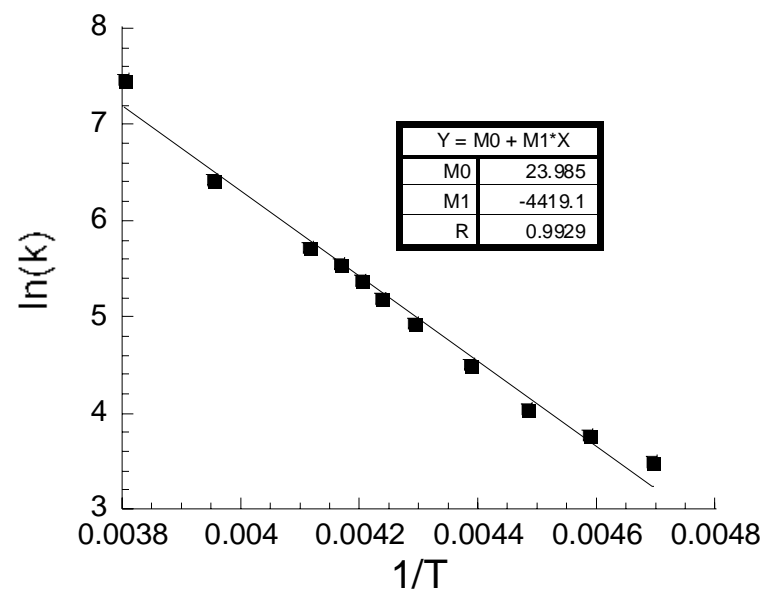

Ea $($ Arrhenius $)=8.82 \mathrm{Kcal} / \mathrm{mol}$ 
Plot of the amide $\mathrm{NH}$ chemical shift (ppm) vs. temperature.

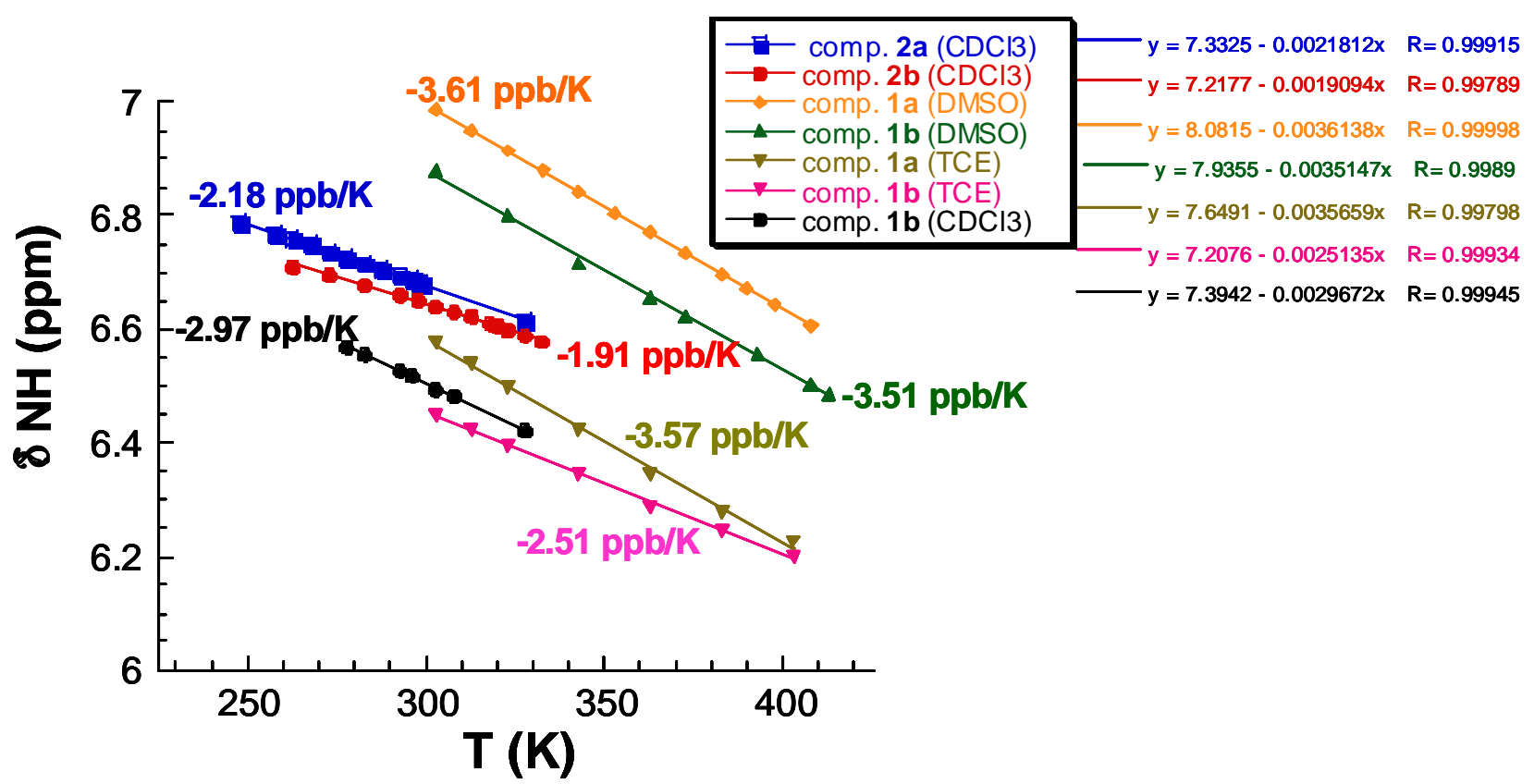

Superimposed ten lowest energy conformations (MMFF94 force field) for $\mathbf{1 a , b - 2 a , b}$ within an energy gap of $3 \mathrm{kcal} / \mathrm{mol}$. Hydrogen atoms have been omitted for clarity.

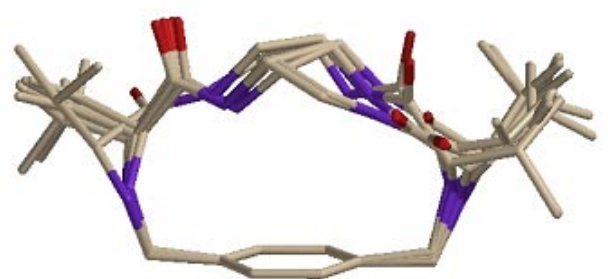

$1 \mathrm{a}$

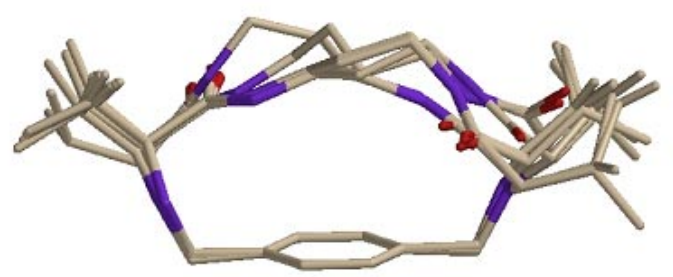

$2 a$

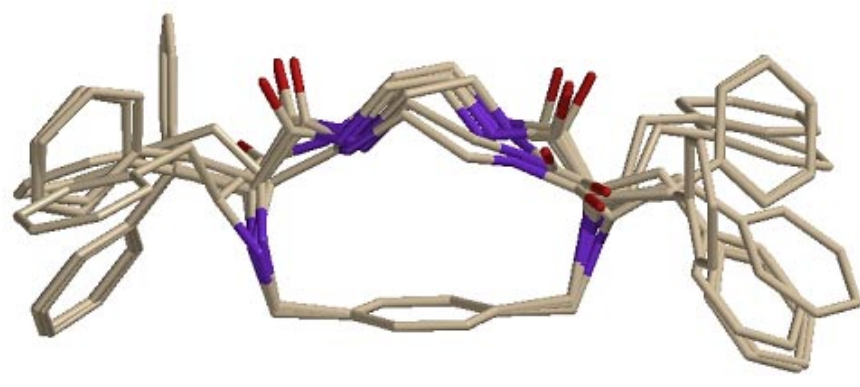

$1 b$

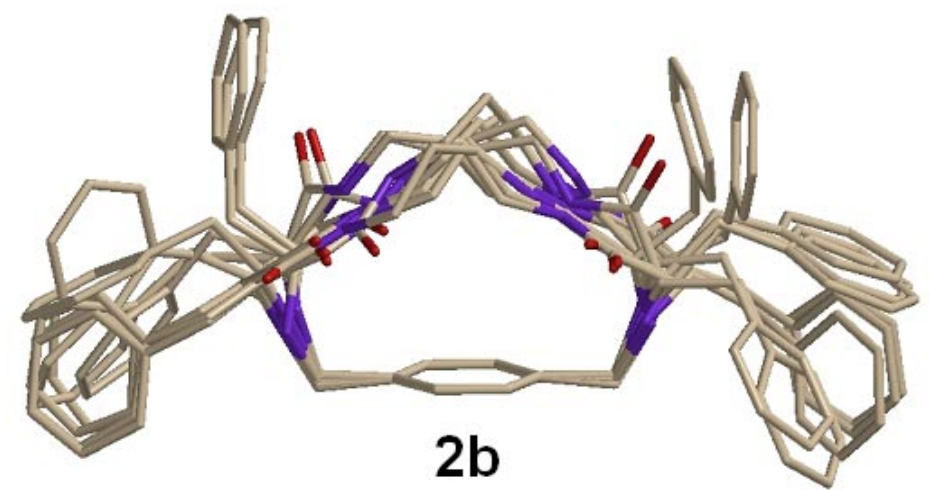


Measured chemical shifts (ppm) for the $p$-diphenylene moiety of $\mathbf{1 a , b - 2 a , b}, \mathbf{b}$, and difference produced by the aromatic side chain $\left[\Delta \delta=\delta\left(\mathrm{R}={ }^{i} \operatorname{Pr}\right)-\delta(\mathrm{R}=\mathrm{Bn})\right]$
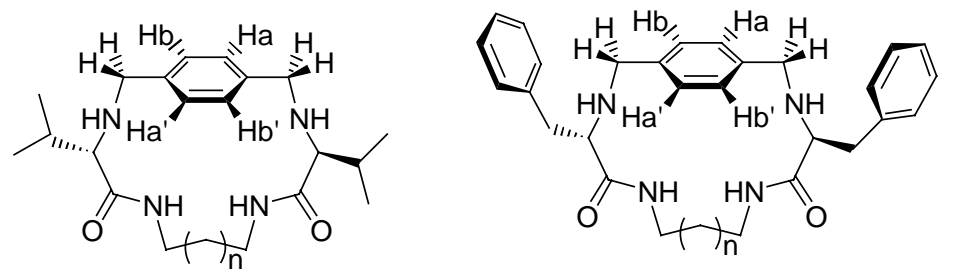

\begin{tabular}{cccccc}
\hline entry & $\mathbf{n}$ & proton & $\mathbf{R}={ }^{i} \mathbf{P r}$ & $\mathbf{R}=\mathbf{B n}$ & $\Delta \boldsymbol{\delta}(\mathbf{p p m})$ \\
\hline 1 & 0 & $\mathrm{Ar}-\mathrm{H}_{\mathrm{a}}$ & 7.062 & 7.003 & 0.059 \\
2 & 0 & $\mathrm{Ar}-\mathrm{H}_{\mathrm{b}}$ & 7.119 & 7.089 & 0.030 \\
3 & 0 & $\mathrm{NCH}_{2}(\mathrm{~A})$ & 3.960 & 3.807 & 0.153 \\
4 & 0 & $\mathrm{NCH}_{2}(\mathrm{~B})$ & 3.283 & 3.123 & 0.160 \\
5 & 1 & $\mathrm{Ar}^{-} \mathrm{H}_{\mathrm{a}}$ & 7.130 & 7.118 & 0.012 \\
6 & 1 & $\mathrm{Ar}^{-} \mathrm{H}_{\mathrm{b}}$ & 7.260 & 7.215 & 0.045 \\
7 & 1 & $\mathrm{NCH}_{2}(\mathrm{~A})$ & 4.180 & 3.923 & 0.257 \\
8 & 1 & $\mathrm{NCH}_{2}(\mathrm{~B})$ & 3.415 & 3.215 & 0.200 \\
\hline
\end{tabular}

Cartesian coordinates for the MMFF94 force field minimum for $1 \mathrm{a}$.

$\begin{array}{lrrc}\mathrm{C} & -3.469208 & -0.421188 & -0.167740 \\ \mathrm{C} & 4.741486 & 1.341507 & 1.332428 \\ \mathrm{C} & 3.366272 & 0.740509 & 0.925751 \\ \mathrm{C} & 2.217239 & 1.727203 & 1.271545 \\ \mathrm{C} & -1.015625 & 1.967239 & 1.592453 \\ \mathrm{C} & -2.843124 & 0.931595 & 0.273010 \\ \mathrm{C} & 1.779434 & -1.618179 & -0.505341 \\ \mathrm{C} & 1.330353 & -2.145889 & 0.710556 \\ \mathrm{C} & -2.389709 & -2.622360 & 0.053833 \\ \mathrm{C} & -0.009750 & -2.495178 & 0.878082 \\ \mathrm{C} & 0.867706 & -1.478958 & -1.562286 \\ \mathrm{C} & -0.926773 & -2.335114 & -0.171722 \\ \mathrm{C} & -0.470474 & -1.839400 & -1.398926 \\ \mathrm{H} & -0.346268 & -2.867798 & 1.842392 \\ \mathrm{H} & 2.014191 & -2.260742 & 1.549118 \\ \mathrm{H} & -2.895294 & -2.882416 & -0.883286 \\ \mathrm{H} & -2.462600 & -3.521286 & 0.680161 \\ \mathrm{H} & -3.057953 & -0.589325 & -1.171295\end{array}$




$\begin{array}{lrrc}\mathrm{H} & -1.459625 & -0.124191 & 1.338531 \\ \mathrm{H} & -3.882065 & -1.887466 & 1.220200 \\ \mathrm{H} & 3.184982 & -0.119263 & 1.583649 \\ \mathrm{C} & 5.864914 & 0.304291 & 1.237183 \\ \mathrm{C} & 3.198578 & -1.131653 & -0.664627 \\ \mathrm{~N} & -1.746628 & 0.818893 & 1.094727 \\ \mathrm{~N} & -3.056335 & -1.519348 & 0.752991 \\ \mathrm{~N} & 1.152786 & 1.699585 & 0.402985 \\ \mathrm{O} & -3.259445 & 2.016556 & -0.128036 \\ \mathrm{O} & 2.232651 & 2.436600 & 2.275772 \\ \mathrm{C} & -0.022141 & 2.533417 & 0.571213 \\ \mathrm{~N} & 3.292480 & 0.323013 & -0.503250 \\ \mathrm{H} & 3.529388 & -1.389130 & -1.678360 \\ \mathrm{H} & -0.509964 & 1.657013 & 2.512192 \\ \mathrm{H} & 3.881836 & -1.665588 & 0.006012 \\ \mathrm{H} & 4.128189 & 0.645828 & -0.986893 \\ \mathrm{H} & 1.221100 & 1.050568 & -0.375290 \\ \mathrm{H} & -1.159210 & -1.701324 & -2.228897 \\ \mathrm{H} & 1.194200 & -1.059753 & -2.512192 \\ \mathrm{C} & 5.122513 & 2.596146 & 0.535446 \\ \mathrm{H} & 0.312744 & 3.521271 & 0.905838 \\ \mathrm{H} & -0.483963 & 2.645584 & -0.415421 \\ \mathrm{C} & -5.009567 & -0.282394 & -0.324310 \\ \mathrm{C} & -5.737061 & 0.013504 & 0.993103 \\ \mathrm{C} & -5.624557 & -1.516556 & -0.992798 \\ \mathrm{H} & -1.745087 & 2.743805 & 1.850540 \\ \mathrm{H} & -5.202103 & 0.568222 & -0.992371 \\ \mathrm{H} & 4.677200 & 1.642334 & 2.386124 \\ \mathrm{H} & 6.800079 & 0.713211 & 1.634308 \\ \mathrm{H} & 6.050308 & -0.002823 & 0.202103 \\ \mathrm{H} & 5.618484 & -0.589400 & 1.820129 \\ \mathrm{H} & 5.298309 & 2.375549 & -0.521805 \\ \mathrm{H} & 6.043488 & 3.033630 & 0.935074 \\ \mathrm{H} & 4.343582 & 3.361664 & 0.598251 \\ \mathrm{H} & -5.328445 & 0.900620 & 1.485200 \\ \mathrm{H} & -5.675980 & -0.824173 & 1.694870 \\ \mathrm{H} & -6.800095 & 0.204956 & 0.804504 \\ \mathrm{H} & -5.131927 & -1.727173 & -1.947632 \\ \mathrm{H} & -6.687851 & -1.352020 & -1.197556 \\ \mathrm{H} & -5.542664 & -2.406555 & -0.360580\end{array}$

Cartesian coordinates for the MMFF94 force field minimum for $1 \mathrm{~b}$.
$\begin{array}{llll}C & 2.643356 & 1.973587 & -0.713226\end{array}$
$\begin{array}{llll}\text { C } & -5.301208 & 0.656174 & 1.902542\end{array}$
$\begin{array}{llll}\text { C } & -3.842377 & 0.574615 & 1.396469\end{array}$
C $-\begin{array}{llll}-3.232132 & 1.997803 & 1.400436\end{array}$
$\begin{array}{llll}\text { C } & -0.697433 & 3.667572 & 0.153259\end{array}$
C $\quad 1.688416 \quad 3.041183-0.123749$
C $-1.410294-1.301544 \quad 0.593018$ 


\begin{tabular}{|c|c|c|c|}
\hline $\mathrm{C}$ & -1.558868 & -1.019440 & -0.770325 \\
\hline $\mathrm{C}$ & 2.001297 & -0.138855 & -1.797012 \\
\hline $\mathrm{C}$ & -0.450974 & -0.674072 & -1.543854 \\
\hline $\mathrm{C}$ & -0.126419 & -1.273300 & 1.156128 \\
\hline $\mathrm{C}$ & 0.828934 & -0.618958 & -0.974792 \\
\hline $\mathrm{C}$ & 0.982773 & -0.941391 & 0.378189 \\
\hline $\mathrm{H}$ & -0.591812 & -0.418945 & -2.592484 \\
\hline $\mathrm{H}$ & -2.542313 & -1.036163 & -1.232376 \\
\hline $\mathrm{H}$ & 2.946700 & -0.536819 & -1.411163 \\
\hline $\mathrm{H}$ & 1.899658 & -0.559631 & -2.805634 \\
\hline $\mathrm{H}$ & 2.795151 & 1.249573 & 0.096771 \\
\hline $\mathrm{H}$ & 0.128433 & 2.011292 & -0.945709 \\
\hline $\mathrm{H}$ & -3.852966 & 0.271988 & 0.341797 \\
\hline $\mathrm{H}$ & -5.316055 & 1.028198 & 2.936554 \\
\hline $\mathrm{C}$ & -2.614044 & -1.555267 & 1.468384 \\
\hline $\mathrm{C}$ & 5.061462 & 1.670044 & -1.496643 \\
\hline $\mathrm{N}$ & 0.354034 & 2.822586 & -0.376419 \\
\hline $\mathrm{N}$ & -1.873596 & 2.040756 & 1.612061 \\
\hline $\mathrm{O}$ & 2.083481 & 3.972366 & 0.576035 \\
\hline $\mathrm{O}$ & -3.894424 & 3.006317 & 1.163544 \\
\hline $\mathrm{C}$ & -1.107971 & 3.271805 & 1.576859 \\
\hline $\mathrm{N}$ & -2.997162 & -0.349686 & 2.214508 \\
\hline $\mathrm{H}$ & -2.346085 & -2.336914 & 2.191116 \\
\hline $\mathrm{H}$ & -1.544617 & 3.603912 & -0.536560 \\
\hline $\mathrm{H}$ & -3.447617 & -1.969833 & 0.890503 \\
\hline $\mathrm{H}$ & -3.546661 & -0.645355 & 3.020676 \\
\hline $\mathrm{H}$ & -1.412704 & 1.150085 & 1.775009 \\
\hline $\mathrm{H}$ & 1.964340 & -0.908081 & 0.845551 \\
\hline $\mathrm{H}$ & 0.007751 & -1.481888 & 2.216003 \\
\hline $\mathrm{H}$ & -5.863525 & 1.399887 & 1.322586 \\
\hline $\mathrm{C}$ & 7.007706 & -0.162857 & -2.343400 \\
\hline $\mathrm{C}$ & 5.662643 & 0.795547 & -0.579956 \\
\hline $\mathrm{C}$ & 5.455400 & 1.620743 & -2.841522 \\
\hline $\mathrm{C}$ & 6.422256 & 0.707474 & -3.261505 \\
\hline $\mathrm{C}$ & 6.630005 & -0.116989 & -1.003113 \\
\hline $\mathrm{H}$ & 5.384750 & 0.824142 & 0.470749 \\
\hline $\mathrm{H}$ & 5.015549 & 2.297516 & -3.570236 \\
\hline $\mathrm{H}$ & 6.724411 & 0.676788 & -4.306046 \\
\hline $\mathrm{H}$ & 7.092682 & -0.790115 & -0.285690 \\
\hline $\mathrm{H}$ & 7.763855 & -0.871231 & -2.671783 \\
\hline $\mathrm{C}$ & -6.048843 & -0.655121 & 1.814972 \\
\hline $\mathrm{C}$ & -7.402222 & -3.104843 & 1.641327 \\
\hline $\mathrm{C}$ & -6.333466 & -1.393768 & 2.971649 \\
\hline $\mathrm{C}$ & -6.460297 & -1.154694 & 0.571152 \\
\hline $\mathrm{C}$ & -7.132278 & -2.374252 & 0.485703 \\
\hline $\mathrm{C}$ & -7.005966 & -2.613480 & 2.883240 \\
\hline $\mathrm{H}$ & -6.038406 & -1.023102 & 3.950348 \\
\hline $\mathrm{H}$ & -6.263367 & -0.593002 & -0.339783 \\
\hline $\mathrm{H}$ & -7.449570 & -2.752655 & -0.482559 \\
\hline $\mathrm{H}$ & -7.225082 & -3.179474 & 3.785080 \\
\hline $\mathrm{H}$ & -7.929993 & -4.052917 & 1.573685 \\
\hline
\end{tabular}




$\begin{array}{rrrr}\mathrm{H} & -1.722885 & 4.068665 & 2.009200 \\ \mathrm{H} & -0.232239 & 3.128708 & 2.217773 \\ \mathrm{C} & 3.997528 & 2.642731 & -1.040588 \\ \mathrm{H} & 4.384445 & 3.169724 & -0.157471 \\ \mathrm{H} & 3.853775 & 3.424576 & -1.798935 \\ \mathrm{H} & -0.336197 & 4.701874 & 0.157303 \\ \mathrm{~N} & 2.015320 & 1.325272 & -1.905640 \\ \mathrm{H} & 2.571808 & 1.569229 & -2.724792\end{array}$

Cartesian coordinates for the MMFF94 force field minimum for 2a.

$\begin{array}{lrrc}\mathrm{C} & -3.451691 & -1.528168 & 0.983444 \\ \mathrm{C} & 5.173080 & 1.137924 & -1.000244 \\ \mathrm{C} & 4.450638 & -0.155029 & -0.525894 \\ \mathrm{C} & 3.584152 & 0.094360 & 0.727386 \\ \mathrm{C} & -0.760925 & 1.036209 & 0.110443 \\ \mathrm{C} & -2.735565 & -0.425873 & 0.172119 \\ \mathrm{C} & 1.994644 & -2.441238 & -0.898956 \\ \mathrm{C} & 1.834671 & -2.877426 & 0.420715 \\ \mathrm{C} & -1.931610 & -3.431488 & 0.635986 \\ \mathrm{C} & 0.564087 & -3.177505 & 0.919403 \\ \mathrm{C} & 0.855148 & -2.285568 & -1.701614 \\ \mathrm{C} & -0.571960 & -3.055176 & 0.105881 \\ \mathrm{C} & -0.414230 & -2.593613 & -1.206451 \\ \mathrm{H} & 0.463913 & -3.516769 & 1.947998 \\ \mathrm{H} & 2.697600 & -2.985214 & 1.074814 \\ \mathrm{H} & -2.631607 & -3.637192 & -0.183823 \\ \mathrm{H} & -1.819580 & -4.395676 & 1.150192 \\ \mathrm{H} & -4.083694 & -2.091614 & 0.284286 \\ \mathrm{H} & -1.168777 & -0.624176 & 1.393784 \\ \mathrm{H} & -2.958160 & -2.992966 & 2.318909 \\ \mathrm{H} & 5.226456 & -0.880981 & -0.250748 \\ \mathrm{C} & 4.244232 & 2.262360 & -1.468765 \\ \mathrm{C} & 3.364777 & -2.155441 & -1.459076 \\ \mathrm{~N} & -1.599167 & 0.066315 & 0.779984 \\ \mathrm{~N} & -2.461441 & -2.459976 & 1.603775 \\ \mathrm{~N} & 2.335541 & 0.611465 & 0.450516 \\ \mathrm{O} & -3.181152 & -0.002014 & -0.890747 \\ \mathrm{O} & 4.004562 & -0.105881 & 1.865005 \\ \mathrm{C} & 1.366150 & 0.815216 & 1.504456 \\ \mathrm{~N} & 3.627029 & -0.720276 & -1.638016 \\ \mathrm{H} & 3.413239 & -2.634293 & -2.446991 \\ \mathrm{C} & 0.214951 & 1.713531 & 1.066284 \\ \mathrm{H} & 4.143677 & -2.658020 & -0.871475 \\ \mathrm{H} & 4.171692 & -0.621857 & -2.495377 \\ \mathrm{H} & 2.012146 & 0.343567 & -0.477295 \\ \mathrm{H} & -1.279953 & -2.467896 & -1.854340 \\ \mathrm{H} & 0.953949 & -1.930283 & -2.724625 \\ \mathrm{C} & 6.129822 & 1.670654 & 0.071793 \\ \mathrm{H} & 1.017075 & -0.164413 & 1.845596\end{array}$




$\begin{array}{rrrr}\text { H } & 1.885010 & 1.283798 & 2.348953 \\ \mathrm{C} & -4.384384 & -0.933929 & 2.075562 \\ \mathrm{C} & -5.495087 & -0.076904 & 1.458725 \\ \mathrm{C} & -3.668518 & -0.139145 & 3.173050 \\ \mathrm{H} & -1.416260 & 1.794983 & -0.331589 \\ \mathrm{H} & -4.883057 & -1.780518 & 2.569565 \\ \mathrm{H} & 5.796066 & 0.860474 & -1.862976 \\ \mathrm{H} & 3.546356 & 1.917786 & -2.237656 \\ \mathrm{H} & 4.829803 & 3.080017 & -1.904541 \\ \mathrm{H} & 3.666061 & 2.684738 & -0.641418 \\ \mathrm{H} & 6.817978 & 0.887512 & 0.405426 \\ \mathrm{H} & 5.589386 & 2.047424 & 0.947006 \\ \mathrm{H} & 6.732391 & 2.494644 & -0.324997 \\ \mathrm{H} & -6.034988 & -0.633881 & 0.686432 \\ \mathrm{H} & -5.098465 & 0.838593 & 1.008500 \\ \mathrm{H} & -6.222733 & 0.218185 & 2.223190 \\ \mathrm{H} & -4.375671 & 0.132278 & 3.965363 \\ \mathrm{H} & -3.240921 & 0.792175 & 2.789108 \\ \mathrm{H} & -2.868027 & -0.722107 & 3.637878 \\ \mathrm{H} & -0.246338 & 0.535217 & -0.715942 \\ \mathrm{H} & -0.329788 & 2.050385 & 1.956497 \\ \mathrm{H} & 0.623703 & 2.608200 & 0.579971\end{array}$

Cartesian coordinates for the MMFF94 force field minimum for 2a.

$\begin{array}{lrrr}\mathrm{C} & -3.856384 & -0.327194 & -0.487015 \\ \mathrm{C} & 4.903046 & 2.122253 & 0.176514 \\ \mathrm{C} & 4.146362 & 0.790436 & 0.376694 \\ \mathrm{C} & 2.961761 & 1.076950 & 1.312744 \\ \mathrm{C} & -0.971344 & 2.155930 & -0.552475 \\ \mathrm{C} & -2.897064 & 0.724731 & -1.067841 \\ \mathrm{C} & 1.850525 & -1.426758 & -0.684921 \\ \mathrm{C} & 1.306778 & -1.831238 & 0.539459 \\ \mathrm{C} & -2.378815 & -2.283432 & -0.322693 \\ \mathrm{C} & -0.061890 & -2.087341 & 0.657639 \\ \mathrm{C} & 0.993347 & -1.261444 & -1.781540 \\ \mathrm{C} & -0.913589 & -1.954956 & -0.448761 \\ \mathrm{C} & -0.374115 & -1.527451 & -1.667435 \\ \mathrm{H} & -0.461777 & -2.402176 & 1.618591 \\ \mathrm{H} & 1.944077 & -1.948578 & 1.414000 \\ \mathrm{H} & -2.821823 & -2.499130 & -1.303116 \\ \mathrm{H} & -2.451279 & -3.230179 & 0.228394 \\ \mathrm{H} & -4.302200 & -0.882355 & -1.322403 \\ \mathrm{H} & -1.794693 & 0.548096 & 0.584976 \\ \mathrm{H} & -3.852386 & -1.765900 & 0.951645 \\ \mathrm{H} & 4.806641 & 0.067169 & 0.872009 \\ \mathrm{H} & 4.275055 & 2.841003 & -0.368011 \\ \mathrm{C} & 3.331207 & -1.186890 & -0.833466 \\ \mathrm{C} & -6.113510 & -0.448029 & 0.704315 \\ \mathrm{~N} & -2.004028 & 1.230011 & -0.142944\end{array}$




$\begin{array}{rrrr}\mathrm{N} & -3.132751 & -1.265625 & 0.425018 \\ \mathrm{O} & -2.977631 & 1.116013 & -2.229416 \\ \mathrm{O} & 3.013733 & 0.860794 & 2.520370 \\ \mathrm{C} & 0.655197 & 1.910980 & 1.399872 \\ \mathrm{~N} & 3.672546 & 0.239624 & -0.932068 \\ \mathrm{H} & 3.644135 & -1.674362 & -1.766495 \\ \mathrm{H} & -0.256454 & 1.620010 & -1.183777 \\ \mathrm{H} & 3.897446 & -1.702866 & -0.048141 \\ \mathrm{H} & 4.465607 & 0.305939 & -1.573624 \\ \mathrm{H} & -1.014023 & -1.395203 & -2.537415 \\ \mathrm{H} & 1.391541 & -0.931427 & -2.739044 \\ \mathrm{H} & 5.096024 & 2.592560 & 1.150726 \\ \mathrm{C} & -8.221100 & -2.114792 & 1.502365 \\ \mathrm{C} & -6.264465 & -0.808136 & 2.049835 \\ \mathrm{C} & -7.035065 & -0.928711 & -0.236038 \\ \mathrm{C} & -8.082900 & -1.759628 & 0.162003 \\ \mathrm{C} & -7.313263 & -1.639771 & 2.445969 \\ \mathrm{H} & -5.564835 & -0.443512 & 2.799835 \\ \mathrm{H} & -6.944260 & -0.655457 & -1.285828 \\ \mathrm{H} & -8.794846 & -2.125977 & -0.573212 \\ \mathrm{H} & -7.423889 & -1.912155 & 3.492493 \\ \mathrm{H} & -9.039764 & -2.759460 & 1.812195 \\ \mathrm{C} & 6.227295 & 1.958588 & -0.536179 \\ \mathrm{C} & 8.672256 & 1.623123 & -1.867233 \\ \mathrm{C} & 7.324600 & 1.390610 & 0.125870 \\ \mathrm{C} & 6.370911 & 2.359772 & -1.871658 \\ \mathrm{C} & 7.587769 & 2.191757 & -2.532883 \\ \mathrm{C} & 8.540421 & 1.224075 & -0.538544 \\ \mathrm{H} & 7.240600 & 1.079346 & 1.164276 \\ \mathrm{H} & 5.535324 & 2.807678 & -2.405426 \\ \mathrm{H} & 7.692123 & 2.508057 & -3.567719 \\ \mathrm{H} & 9.388016 & 0.785721 & -0.017349 \\ \mathrm{H} & 9.620560 & 1.494614 & -2.382080 \\ \mathrm{H} & 0.193176 & 0.946503 & 1.631592 \\ \mathrm{H} & 0.917603 & 2.380249 & 2.355072 \\ \mathrm{C} & -4.965881 & 0.436539 & 0.270050 \\ \mathrm{H} & -5.372131 & 1.235016 & -0.366119 \\ \mathrm{H} & -4.546051 & 0.954559 & 1.142715 \\ & -0.292068 & 2.830887 & 0.635391 \\ \mathrm{H} & -1.060165 & 3.200394 & 1.326019 \\ \mathrm{H} & 1.884872 & 1.665665 & 0.678879 \\ & & 1.395416 & -0.302780\end{array}$

Cartesian coordinates for the transition state of 1a (AM1 level of theory)
C $\quad-3.753006 \quad-1.145905 \quad-0.027710$
$\begin{array}{llll}\text { C } & 1.756882 & 1.851379 & -1.108185\end{array}$
C $\quad-2.986298 \quad 0.111633 \quad 0.493668$ 


\begin{tabular}{|c|c|c|c|}
\hline $\mathrm{H}$ & -2.972198 & 1.031860 & -1.348572 \\
\hline $\mathrm{H}$ & -2.562439 & -1.743805 & -1.551834 \\
\hline $\mathbf{N}$ & -2.731537 & 1.157547 & -0.394333 \\
\hline $\mathbf{N}$ & -2.960800 & -2.140594 & -0.721832 \\
\hline $\mathbf{N}$ & 0.671234 & 2.627075 & -0.698868 \\
\hline & -2.639984 & 0.210342 & 1.684235 \\
\hline $\mathrm{O}$ & 1.676468 & 1.171738 & -2.147507 \\
\hline $\mathrm{N}$ & 3.116959 & 1.052780 & 0.906800 \\
\hline $\mathrm{C}$ & 3.175949 & -0.391479 & 0.808655 \\
\hline $\mathrm{H}$ & 0.744827 & 3.119858 & 0.158859 \\
\hline C & -4.583878 & -1.703445 & 1.152206 \\
\hline $\mathrm{H}$ & 2.408585 & 1.334152 & 1.558609 \\
\hline $\mathrm{C}$ & -1.987717 & -2.962433 & -0.032013 \\
\hline $\mathrm{H}$ & 3.811768 & -0.673767 & -0.080093 \\
\hline $\mathrm{H}$ & 3.709381 & -0.738937 & 1.742416 \\
\hline $\mathrm{C}$ & -0.599777 & -2.407394 & 0.136551 \\
\hline $\mathrm{H}$ & -1.925507 & -3.923431 & -0.623505 \\
\hline $\mathrm{H}$ & -2.380020 & -3.211639 & 0.995789 \\
\hline $\mathrm{C}$ & 0.502228 & -3.145706 & 0.585709 \\
\hline $\mathrm{C}$ & -0.412460 & -1.044189 & -0.077637 \\
\hline $\mathrm{C}$ & 0.735046 & -0.408417 & 0.355698 \\
\hline $\mathrm{H}$ & -1.134827 & -0.435120 & -0.625931 \\
\hline $\mathrm{C}$ & 1.873932 & -1.136139 & 0.689117 \\
\hline $\mathrm{H}$ & 0.685074 & 0.677704 & 0.459427 \\
\hline $\mathrm{H}$ & 0.401108 & -4.227737 & 0.759583 \\
\hline $\mathrm{C}$ & 1.742188 & -2.526306 & 0.796356 \\
\hline $\mathrm{H}$ & 2.614746 & -3.138992 & 1.066010 \\
\hline $\mathrm{H}$ & -4.498322 & -0.781769 & -0.804611 \\
\hline $\mathrm{H}$ & -3.887161 & -1.885696 & 2.018005 \\
\hline $\mathrm{C}$ & -5.280426 & -2.995773 & 0.782959 \\
\hline $\mathrm{C}$ & -5.615204 & -0.673035 & 1.573303 \\
\hline $\mathrm{H}$ & -4.546967 & -3.827759 & 0.654007 \\
\hline $\mathrm{H}$ & -5.850174 & -2.876740 & -0.169983 \\
\hline $\mathrm{H}$ & -5.996933 & -3.283554 & 1.591278 \\
\hline $\mathrm{H}$ & -5.119507 & 0.282944 & 1.868759 \\
\hline $\mathrm{H}$ & -6.192505 & -1.055313 & 2.449646 \\
\hline $\mathrm{H}$ & -6.331787 & -0.466736 & 0.741852 \\
\hline $\mathrm{C}$ & 3.072998 & 1.899506 & -0.268661 \\
\hline $\mathrm{C}$ & 4.253464 & 1.692398 & -1.246735 \\
\hline $\mathrm{H}$ & 3.170303 & 2.956177 & 0.137161 \\
\hline $\mathrm{H}$ & 4.077942 & 0.736481 & -1.815140 \\
\hline $\mathrm{C}$ & 5.576920 & 1.608917 & -0.514725 \\
\hline $\mathrm{C}$ & 4.294205 & 2.841064 & -2.236923 \\
\hline $\mathrm{H}$ & 6.416977 & 1.625229 & -1.250610 \\
\hline $\mathrm{H}$ & 5.650604 & 0.665939 & 0.078674 \\
\hline $\mathrm{H}$ & 5.696976 & 2.474136 & 0.181244 \\
\hline $\mathrm{H}$ & 5.122375 & 2.679000 & -2.967422 \\
\hline $\mathrm{H}$ & 4.471390 & 3.810837 & -1.712906 \\
\hline $\mathrm{H}$ & 3.333939 & 2.906937 & -2.803665 \\
\hline $\mathrm{C}$ & -1.751282 & 2.174057 & -0.100327 \\
\hline $\mathrm{C}$ & -0.660019 & 2.382629 & -1.196304 \\
\hline
\end{tabular}



$\begin{array}{llll}\mathrm{H} & -1.274826 & 1.927963 & 0.892868\end{array}$
$\begin{array}{llll}\mathrm{H} & -2.298691 & 3.152863 & 0.020889\end{array}$
$\begin{array}{llll}\mathrm{H} & -0.619919 & 1.494476 & -1.891052\end{array}$
$\begin{array}{llll}\mathrm{H} & -0.966873 & 3.278580 & -1.810043\end{array}$ 